\title{
« Métamorphoses du poème »
}

In : Failles fertiles du poème, Tarabuste, 1999, p. 42-48. Présenté par

Hervé Sanson

Jamel-Eddine Bencheikh

Hervé Sanson (éd.)

\section{OpenEdition}

\section{Journals}

Édition électronique

URL : https://journals.openedition.org/coma/934

DOI : $10.4000 /$ coma.934

ISSN : 2275-1742

Éditeur

Institut des textes \& manuscrits modernes (ITEM)

\section{Référence électronique}

Jamel-Eddine Bencheikh, « « Métamorphoses du poème » », Continents manuscrits [En ligne], 9 | 2017 mis en ligne le 15 octobre 2017, consulté le 12 janvier 2023. URL : http://journals.openedition.org/ coma/934 ; DOI : https://doi.org/10.4000/coma.934

Ce document a été généré automatiquement le 12 janvier 2023.

\section{(i) 8.}

Creative Commons - Attribution - Pas d'Utilisation Commerciale - Pas de Modification 4.0 International - CC BY-NC-ND 4.0

https://creativecommons.org/licenses/by-nc-nd/4.0/ 


\title{
« Métamorphoses du poème »
}

\author{
In : Failles fertiles du poème, Tarabuste, 1999, p. 42-48. Présenté par \\ Hervé Sanson
}

Jamel-Eddine Bencheikh

Hervé Sanson (éd.)

\section{NOTE DE L'ÉDITEUR}

«Je rêve d'une page qui paraîtrait à l'œil un dessin de mots et de lignes, mais qui, examiné à la loupe, démentirait cet ordonnancement, révélerait en chaque lettre un fourmillement de signes-mouvements, de courbes-temps, de traits-lieux. » Ces lignes sont extraites de la fin du texte " Métamorphoses du poème ", du poète et critique algérien Jamel-Eddine Bencheikh (1930-2005), texte consacré à la genèse du poème. Pour qui traque la vérité du poème depuis la position du critique, le constat est on ne peut plus évident : l'explication de la genèse d'un poème est une gageure. Entre le poète et son exégète, circulent deux langages bien souvent hétérogènes. Ainsi que le rappelait Habib Tengour dans les entretiens qu'il m'accorda sous le titre La Trace et l'écho (2012), « [N]e rentre véritablement dans le poème que celui qui est animé d'une intention droite. Celui qui veut y entrer, celui-là fera l'effort nécessaire en amitié. » Il y faut l'empathie, l'acceptation d'une certaine obscurité préalable avant de se laisser gagner par le poème. Les outils clés-en-mains prétendent expliquer mais n'atteignent rien d'authentiquement profond des mécanismes à l'origine de ce que l'on nomme " poème ». C'est par le biais entre autres d'un lexique pictural que Bencheikh débusque la vérité du poème, son alchimie secrète. Le maitre-mot est " échanges », que Bencheikh utilise dans l'extrait que nous donnons à lire. Échanges tant avec le critique qui doit se dépourvoir de ses bagages théoriques, et accepter le risque du poème, qu'avec le poème lui-même qui, dans la phase de reprise et de correction, dialogue avec son créateur, et instaure une série de mouvements, de déplacements, attestant que le poème in fine se veut (selon une obstétrique active) un vecteur d'énergies, un rythme couronné qui doit marquer au corps le lecteur. C'est ainsi que Bencheikh le conçoit comme un trajet, un cheminement vers une réserve de sens, que l'avancée forge en 
partie. Dans la revue qui est la nôtre, spécialisée dans la génétique des textes, la question est la suivante : que peut notre discipline face au mystère du poème ? Au-delà de ce que peut apporter la génétique en suivant l'évolution des avant-textes de l'œuvre, l'extrait qui suit, fruit de la parole d'un poète, nous donne quelques pistes de réflexion fructueuses, à nous autres « techniciens » du texte littéraire.

Hervé Sanson

1 (...) Le poème est un lieu d'échanges. Rien ne le montre mieux que la correction d'un texte, ce travail que l'écriture vous impose de faire sur elle-même. Appel de nécessité : de l'ébauche au texte final se dessine une trajectoire du franchissement.

2 Si je relis des lignes écrites la veille ou quelque temps auparavant, une chose m'apparaît immédiatement, il y a des mots qui n'y sont pas à leur place. Je ne parle pas des facilités, des futilités ou des inadvertances, je désigne les mots qui, pour différentes raisons, ne s'inscrivent pas dans l'espace du poème, ne font pas partie de son soubassement. Chaque rature provoque un soulagement, comme si le poème s'allégeait d'un .poids mort. Si un mot devient une charge inutile, ce n'est pas qu'il soit moins poétique qu'un autre, qu'il contrevienne au rythme ou brouille l'image (cela peut compter aussi, mais si peu et ne s'inscrit qu'en surface), s'il devient corps-mort, c'est parce qu'il gît sur la trajectoire vers le sens, qu'il ne transmet pas le mouvement. Cellule vide, il ne communique rien de ce qu'il a reçu, il s'oppose à l'avancée, il interrompt l'investigation. L'énoncé doit le contourner. Le supprimer ou le remplacer rétablit proprement le circuit.

3 La correction relève ainsi d'une mise au jour du processus d'écriture. En désignant les ruptures, elle repère une continuité ; en prononçant des exclusions, elle reconnaît et dessine les limites de l'espace signifiant. L'opacité du mot corrigé s'opposait moins à l'intention du poète qu'à la fonction du poème. On comprend alors pourquoi, même au cœur des surprises les plus fulgurantes, les mots s'ajustent les uns aux autres, se conviennent, accourent hors des habitudes du langage pour se prêter main-forte, assurer les relais du sens. Ainsi se mesure un parcours, ainsi se débusquent les significations. Tout ce qui fait opposition à cette entreprise doit être supprimé. Tout usage artificiel de la langue qui n'y contribuerait pas relève de l'effet littéraire.

Certains textes se constituent immédiatement. On dit qu'on les jette sur le papier tout d'un trait. Ils se déconstituent aussi spontanément lors de la correction. La plume encadre un, deux, dix mots ressentis comme contraires à un sens abstrait dont on a un sentiment aussi net qu'inexplicable. Se dessinent alors les contours d'un nouveau poème qui émerge peu à peu du texte comme une île volcanique surgit à la lumière au cours de la gésine des profondeurs. Surprenante métamorphose que l'apparition de ce corps immergé dont on s'aperçoit qu'il existait de toujours, qu'il suffisait d'en découvrir le dessin pour lui donner vie. Le tissu trop lâche du premier texte n'était en réalité qu'un filet lancé pour capturer le second dans ses mailles.

5 Mais le poème sait aussi prononcer son achèvement. A un moment donné, il s'avère impossible de rien lui retrancher ou lui ajouter. Sa métamorphose est terminée. Il a trouvé sa signification. La clôture se prononce-t-elle par la juxtaposition exacte du texte réalisé au sens rêvé ? Cela serait trop simple de le croire ou se rapprocherait, sans le vouloir, d'une vieille théorie du sens qui prononce l'excellence de l'énoncé pour son adéquation à l'objet qu'il vise. Les corrections ne sont pas dictées par la comparaison de l'ébauche avec un tout achevé, déjà constitué dans la conscience ou ailleurs, désiré par 
l'écriture pour qu'elle s'y ajuste. Il n'existe pas d'idée de poème parfait vers lequel on tend. L'achèvement ne consacre donc pas une réussite, il ne salue pas un dit enfin efficace. Il marque la fin d'un certain travail sur le sens. Avec ces moyens-là, le poème ne saurait aller plus loin ou autrement. Il s'immobilise où l'a conduit son mouvement. Il n'y a pas épuisement du sens, mais suspension d'un cheminement. État provisoire d'une métamorphose continue. Chaque poème vit sa propre aventure, et se propose aussi comme une halte dans l'aventure générale d'une écriture. Il est à la fois totalité de soi et moment d'un tout.

Il y a dans toute métamorphose poétique une énergie et une tragédie. La première préside au devenir. L'écriture jonche sa route de défroques dont elle a dénoncé l'usure. Elle prend à partie les conventions, bafoue les codes, déchiffre de nouveau les signes. Devant l'inconnu, elle a l'entêtement et l'audace dont ne dispose pas toujours la science. Elle ne saurait d'ailleurs être réduite à l'aveu d'un progrès qu'accompagne la désespérance. Si elle ne peut transformer les hommes, elle peuple leur langue d'une présence irréductible qui témoignera malgré tout de leur être. C'est dans la langue qu'elle mène son essentiel combat moral, en elle quelle inscrit les rendez-vous à prendre avec l'avenir. Elle est rebelle à tout ce qui réduit ou torture l'existence. Armée d'intuitions majeures, elle précède toute conscience et toute science sur les chemins où s'imagine un monde à naître. Elle forge sans fatigue des vérités qu'elle sent palpiter dans ses rythmes, ses images, ses phrases. Elle invente un alphabet qui épelle peu à peu la substance d'être. Perse l'a superbement écrit : «Si la poésie n'est pas, comme on l'a dit, le réel absolu, elle en est bien la plus proche convoitise et la proche appréhension, à cette limite extrême de complicité où le réel dans le poème semble s'informer lui-même. " (Discours de Stockholm).

7 Et tant pis si elle n'est pas écoutée, si elle reste en marge de culture comme une sentinelle muette. Tant pis, car elle n'a pour mission ni de plaire ni de se prostituer, elle est au-delà de tout imaginaire actuel; il faut, pour la percevoir, entrer soi-même en métamorphose, corps, âme,- esprit. Sa tragédie n'est pas de n'être point comprise ou de l'être trop. Elle n'a que faire des jeux de scène et des accommodements de fortune. Sa tragédie est inscrite dans sa fonction. Dès lors qu'elle a cessé d'orner pour bouleverser, de bien dire pour imaginer des langages promis, il n'est pas sûr qu'elle se maintienne en un si périlleux exercice, qu'elle ne prononce pas elle-même l'écart définitif entre l'homme d'acquiescement et l'être de refus.

8 La haute vertu du poème pourrait d'ailleurs n'être pas le privilège de la créature humaine. La pierre ne pense pas son éternité, pas plus que l'animal sa mort. L'homme pourrait finir par envier l'immobile sérénité de l'une et l'instinctive ignorance de l'autre. Il ne confierait plus à la parole qu'une existence de néant d'où seraient exclus et l'inquiétude et l'amour, ces sources majeures des métamorphoses.

INDEX

Mots-clés : Algérie, poésie, genèse 